\title{
Spectral Efficient Pulse Shaping for Impulse Radio Ultra Wideband Communications
}

\author{
${ }^{* 1}$ Devika Menon M.K, ${ }^{2}$ Joseph Rodrigues, ${ }^{3}$ Linu Lonappan, ${ }^{4}$ Lucy J.Gudino \\ ${ }_{1,2,3}$ CHRIST (Deemed to be University), Bengaluru, India \\ ${ }^{4}$ Birla Institute of Technology and Science, Pilani, India \\ Email:devika.menon@christuniversity.in, joseph.rodrigues@christuniversity.in, \\ linu.lonappan@christuniversity.in,lucy.gudino@pilani.bits-pilani.ac.in
}

Received: 06th December 2019, Accepted: 20th January 2020, Published: 30th April 2020

\begin{abstract}
Efficient pulse shaping techniques are required in UWB communication systems to fit the Federal Communication Commission (FCC) mask with reduced interference with other narrow band systems. We propose a pulse shaping technique to fit the FCC mask efficiently by employing our proposed band pass filter. The UWB pulse shape is spectrally modified and analyzed to prove its ability in achieving higher spectral utilization efficiency without employing any optimization techniques as in literature. The proposed band pass filter for pulse shaping has features of sharp transition, desired stop band attenuation and expression for filter coefficients which enables flexible spectral shaping to fit any FCC mask efficiently.
\end{abstract}

Keywords

Impulse Radio, Monopulse, Spectral Pulse Shaping, Power Efficiency, Power Spectral Density.

\section{Introduction}

Ultra-wide band systems have a fractional bandwidth exceeding 0.20 or an absolute bandwidth exceeding $500 \mathrm{MHz}$ as defined by FCC (Y.Rahayu et al., 2008; R.K.Dokania et al., 2011; HaoluXie et al., 2008; Hyunseok Kim et al., 2005). The FCC has standardized the spectral shape and mandated power spectral density (PSD) of $-41 \mathrm{dBm} / \mathrm{MHz}$ for UWB devices for preventing interference with other communication technologies. For UWB communication schemes, efficient pulse shapers are needed for the spectrum of UWB pulses within the FCC mask and high energy effectiveness with negligible interference with other narrow band systems. The pulse shaper designs should be such that the bandwidth and power permitted by the spectral mask of the FCC should be used optimally. The baseband UWB systems can be single-band or multi-band. UWB radio systems transmitter and receiver use single-band or multi-band pulses. In singleband (SB) UWB systems, only one transmission frequency band is used and two or more frequency bands are used in multi-band (MB) UWB systems, each with a bandwidth of at least $500 \mathrm{MHz}$ (Y.Rahayu et al., 2008; G.Zhang et al., 2011).The SB-based UWB systems are also referred to as carrier-free or Impulse Radio (IR) UWB communication systems as the signal generated is very short, low duty cycle, baseband pulse of required shape and duration(M.Z.Win et al., 1998; S.M.Ekome et al., 2011; B.A. Lagovsky et al., 2016). These pulses have quick rise and fall times, resulting in wideband spectra. Then such systems have low system complexity, reduced expenses due to direct transmission and reception of pulsed signals and also owing to the need for the least radio frequency equipment at their front ends as compared to standard narrowband radio systems (B.Parr et al., 2013; L.Li et al., 2011) . MB UWB systems implementation can be carrier less or carrier based (Hongsan Sheng et al., 2003; M.Sablatash et al., 2006). The carrier-based UWB scheme is known as multiplexing UWB orthogonal frequency division (UWB-OFDM) where communication methods are used to divide the bandwidth into sub-bands. The ultra-short pulses employed in UWB systems have the advantages of high data rates, superior localization properties, low complexity receivers, multi-path immunity, jamming resistant, and very excellent time-domain resolution that are crucial for location and tracking applications (I.I.Immoreev et al., 2002; H.Werfelli et al., 2013).UWB transmitter does not require mixing stage for the signal propagation since they produce very short timedomain pulses of nanosecond duration unlike narrowband. Since the permissible power for data transmission is very low these pulses are designed with very low energy. The UWB has applications in wireless communications, radar imaging, vehicle radar, high-data communications, automotive radar, ground penetrating radar, radio frequency identification, in-wall imaging, consumer electronics and PCs as well as wireless sensor networks.

The contents of thispaper include review of UWB pulse shaping techniques, expression of filter coefficients of a novel FIR filter for UWB pulse shaping, simulations and results of UWB pulse shaper and conclusion.

Review of UWB Pulse Shaping Techniques

The UWB pulse waveform is a function which should meet the spectral mask regulatory requirements. Since the transmission power spectrum in UWB devices is strictly restricted by the statutory FCC spectral mask, the pulse 
shape layout is critical to achieving ideal spectrum usage, spectral mask compatibility and coexistence with other wireless facilities. UWB transmitter (TX) employs few methods to generate desired UWB signal (Y.Wang et al., 2008; H.Werfelli et al., 2015). In some UWB TX, filters are employed to shape base band narrow pulses to produce the required UWB signal. It has a drawback of the high cost involved for custom filters and poor in tuning capability once manufactured (Y.Wang et al., 2008). For some TXs, digital controlled synthesis techniques are used to arbitrarily modify the output shape to meet FCC standards. But for precise control of subimpulses, this method needs high speed / resolution, digital-to-analog converters or delay generators. Another technique for generating a required UWB signal is to convert a baseband pulse with a particular form of the envelope used in carrier-based TX. To comply with the FCC regulations, existing carrier-based TXs require additional high-order off-chip or multi-inductor shaping / filtering circuits at the output or delicate pulse shapes, e.g. for TXs are required high-order derivatives of Gaussian pulses.

A range of pulse shapes have been suggested for UWB impulse radio devices, including Gaussian, Laplacian, Rayleigh or Hermitean pulses (J.Joe et al., 2003), to meet the optimal transmission power spectrum as required by the FCC (J.Joe et al., 2003) modified Hermitian monocycles (B.Parr et al., 2003; Hongsan Sheng et al., 2003; M.Ghavami et al., 2002; M.Ghavami et al., 2008), Prolate Spheroidal wave functions (Hongsan Sheng et al.,2003), Gaussian pulse and Gaussian derivatives (Xiliang Luo et al., 2003; L.A.de Ávila et al., 2016). The comparison of most popular pulses with respect to FCC mask and their energy efficiency are presented in (Xiliang Luo et al., 2003) and (B.Parr et al., 2003) . Gaussian pulse is the most common pulse shape used in UWB transmission due to its simplicity. But it has a limitation that, it is not flexible enough to meet the strong requirements of efficient spectral utilization and flexible interference avoidance (X.Liu et al., 2008) . Due to shorter durations than the Hermitian pulses, Prolate Spheroidal pulses are preferable. But the limitation is that they produce strong adjacent channel interferences due to the large sidelobes in the power spectral densities (PSDs). Modified Prolate Spheroidal wave functions are employed to obtain high spectral efficiency and to meet UWB specifications. A novel technique for the construction of UWB pulses is suggested based on the linear combination of Gaussian pulses in (N.C. Beaulieu et al., 2006) .The produced pulses have a flexible property for preventing interference and thus guarantee negligible interference with other existing narrow band (NB) schemes. They also meet the mask criteria of the FCC Power Spectral Density (PSD). An approximate sinc function using Gaussian pulse as the basic function is shown in the pulse shaping technique discussed in (X.Liu et al., 2008). The produced pulse has appealing characteristics such as high spectrum utilization efficiency (SUE), FCC mask compliance, flexible interference prevention, flexible pulse duration to fulfil various data rate demands and very excellent jitter resistant property particularly when timing jitter is comparatively high in multipath setting. Hamming and Hann windowed pulses (N.C. Beaulieu et al., 2006) and sinc based monocycles (N.C. Beaulieu et al., 2008) are the widely used technologies for generating orthogonal UWB pulses in multiple access applications to decrease bit error rates. Under different channel circumstances, the spectrum feature and the bit error rate of the pulse waveforms are discussed in (J.Joe et al., 2003) . Considering additive white Gaussian noise channels, multiple access interference channels and fading multi-path channels, the different channel conditions are realized. The algorithm provided in (Lu Yin et al., 2005) is flexible in developing ultrawideband pulses that meet the demands of the FCC spectral mask and has superior bit error rate performance. A novel pulse design is suggested based on Hermite functions in (M.Ghavami et al., 2007). The pulses meet the multi-access FCC emission boundaries and the appropriate pulses have excellent orthogonality. AWGN noise performance assessment demonstrates that these pulses are robust to pulse jitter and deliver outstanding BER output. (Bo Hu et al., 2005) introduces an algorithm designing two powerful orthogonal pulses restricted to a short time. These pulses power spectra fit the desired frequency mask. Prolate Spheroidal wave functions are another widely employed FCC-compliant orthogonal pulses. The pulse response generated employing pulse shapers can be approximated to the least square sense of Prolate Spheroidal pulses (B.Parr et al., 2003) . Other transfer functions that provide highly extremely impulse responses which are FCC compliant are also presented. The PSD is minimized by distributing energy in frequency range and thus reducing the interference probability with other user systems. Modified Hermitian and Prolate Spheroidal pulses are commonly used in multi-access UWB communications using orthogonal pulses.

FIR filter techniques are extensively employed for pulse shaping. Spatial filters also extensively use FIR filters in antenna array beamforming to obtain a highly directive beam (L.J.Gudino et al., 2008; L.J.Gudino et al., 2009). The designed pulses have higher flexibility and FCC spectral mask compliance attained employing the Parks-McClellan algorithm (E.Baccarelli et al., 2008). However, since the length of the filter and therefore the pulse length is very large, this can lead to overlapping pulses, increased energy consumption, interference and therefore the proposed method is not an optimal solution. The semi-definite programming (SDP) method is used in (M.Rezaii et al., 2010) to reduce the filter length as well as to meet FCC spectral mask specifications. Pulse shaping is done with pulse shapers called band pass filters. But the filtering methods that extend the pulse duration reduce the data rate and system ability. The challenge is therefore to design short-term pulses to decrease power distribution interference in a frequency band from $3.1 \mathrm{GHz}$ to $10.6 \mathrm{GHz}$. The design of the filters is influenced by the selection of pulse form, recipient bandwidth selection, bit error rate and efficiency in 
multi-path propagation settings. In this paper, we described the design of an FIR filter in order to achieve a pulse with optimal power efficiency. We discussed an approach to formulate the magnitude constraint. We applied this approach for shaping monocycle pulse using filters of different length for compliance with required FCC mask spectral emission constraints.

In this paper, a UWB pulse shaping technique is introduced using a FIR band pass filter. This technique is flexible to fit into any FCC mask with good spectral utilization efficiency. A Closed form expression is obtained as a shaping function for this purpose.

\section{Expression for Filter Coefficients}

In this section, an expression for filter coefficients of band pass filter is proposed for spectral shaping of the UWB pulse. The proposed filter possesses sharp transition, low pass band ripple and good stop band attenuation to fit the FCC mask regulations. Its magnitude response can be tailor made to fit any FCC mask requirements. The magnitude response $\mathrm{H}(\mathrm{f})$ of the filter is modelled with well behaved trigonometric functions of frequency. Applying the cosine transformation (L.J.Gudino et al., 2008) to the magnitude function H(f), we obtain an expression for filter coefficients of the band pass filter as,

$$
\begin{aligned}
& h(n)=\frac{\mathrm{A} \delta_{p} \cos \mathrm{k} f_{b}}{\pi\left(U_{p b}^{2}-k^{2}\right)}\left[U_{p b} \sin \mathrm{U}_{p b} f_{p} \cos \mathrm{k} f_{p}-\mathrm{k} \cos \mathrm{U}_{p b} f_{p} \sin \mathrm{k} f_{p}\right] \\
& +\frac{\mathrm{A} \delta_{\mathrm{s}} \mathrm{U}_{\mathrm{pb}}}{2 \pi\left(U_{\mathrm{pb}}^{2}-\mathrm{k}^{2}\right)}\left[\cos \mathrm{U}_{\mathrm{pb}}\left(f_{\mathrm{b}}-f_{\mathrm{z}}\right)-\cos \mathrm{k}\left(f_{\mathrm{b}}-f_{\mathrm{z}}\right)\right] \\
& +\frac{\mathrm{A}}{\mathrm{k}^{2} \pi\left(f_{z}-f_{p}\right)}\left[\cos \mathrm{k}\left(f_{b}-f_{p}\right)-\cos \mathrm{k}\left(f_{b}-f_{z}\right)+\mathrm{k}\left(f_{z}-f_{p}\right) \sin \mathrm{k}\left(f_{b}-f_{p}\right)\right] \\
& +\frac{\mathrm{A}}{\mathrm{k}^{2} \pi\left(f_{z}-f_{p}\right)}\left[\cos \mathrm{k}\left(f_{b}+f_{p}\right)-\cos \mathrm{k}\left(f_{b}+f_{z}\right)-\mathrm{k}\left(f_{z}-f_{p}\right) \sin \mathrm{k}\left(f_{b}+f_{p}\right)\right] \\
& +\frac{\mathrm{A} \delta_{\mathrm{s}}}{2 \pi\left(U_{\mathrm{pb}}^{2}-\mathrm{k}^{2}\right)}\left[\left[U_{\mathrm{pb}} \cos \mathrm{U}_{\mathrm{pb}}\left(\pi-\left(f_{\mathrm{b}}+f_{\mathrm{z}}\right)\right) \cos \mathrm{k} \pi-U_{\mathrm{pb}} \cos \mathrm{k}\left(f_{\mathrm{b}}+f_{\mathrm{z}}\right)\right]\right. \\
& \left.+\mathrm{k} \sin \mathrm{U}_{p b}\left(\pi-\left(f_{b}+f_{z}\right)\right) \sin \mathrm{k} \pi\right]+\frac{2 \mathrm{~A}}{\mathrm{k} \pi} \cos \mathrm{k} f_{b} \sin \mathrm{k} f_{p}
\end{aligned}
$$

where, $n=0,1, \ldots . .(\mathrm{L} / 2)-1$ for $\mathrm{L}$ even $, n=0,1, \ldots . . \mathrm{L}-(1 / 2)$, for $\mathrm{L}$ odd and $\mathrm{k}=\{(\mathrm{L}-1) / 2\}-n$.

The band pass filter design parameters are A the peak gain, $U_{p b}$ controls the shape of magnitude function $\mathrm{H}(\mathrm{f})$ in the pass band, $f_{p}$ is the pass band frequency, $\mathrm{H}(\mathrm{f})=0$ at frequency $f_{\mathrm{z}}$ for the filter design model, $f_{\mathrm{b}}$ is the centre frequency, $\delta_{p}$ is the pass band ripple, $\delta_{\mathrm{s}}$ is the stop band attenuation and $\mathrm{L}$ is the length of the filter.

\section{Simulations and Results}

A UWB band pass filter for pulse shaping is synthesized to fit the FCC mask of $3.1 \mathrm{GHz}$ to $10.6 \mathrm{GHz}$. The designed band pass filter is employed to shape the Gaussian monocycle pulse to obtain a modified pulse with good power efficiency employing filters having length $\mathrm{L}$ of 21 and 37. The filter normalized specifications to fit the FCC mask are centre frequency $f_{\mathrm{b}}$ of $0.6 \pi$, lower cut-off frequency $\mathrm{f}_{\mathrm{L}}$ of $0.2575 \pi$, upper cut-off frequency of $\mathrm{f}_{\mathrm{U}}$ of $0.9425 \pi$ and transition band of $0.018 \pi$. The sampling frequency Fs is $22 \mathrm{GHz}$. We consider a UWB Gaussian monopulse with Tg of 0.186ns. Fig.1 and Fig.2 respectively shows the FCC mask and PSD of the proposed pulse shaping bandpass FIR filter with L of 37 and L of 21. Its impulse response h(n) is shown in Fig.3. For L of 21, it is observed in Fig.2 that side lobe level (SLL) and power efficiency deteriorates. The obtained PSD very closely matches the FCC spectrum and power efficiency of $91.2 \%$ is achieved for L of 37 . The Power efficiency of the proposed method is compared with other methods as shown Table 1. An improvement of $7.2 \%$ in power efficiency is achieved for $\mathrm{L}$ of 37 compared to $\mathrm{L}$ of 21 . It is observed that for the same power efficiency, the length of UWB pulses required for our proposed method is lesser than some methods as inferred from Table 1. In addition, the suggested technique does not use computationally intensive optimization techniques. It is observed from Fig. 1 that the proposed FIR filter has tapering frequency response characteristics which fits into the FCC mask efficiently. It possesses good first null to last null ratio of 0.852 and flat pass band is achieved that increases spectral utilization efficiency. Other advantages of the proposed pulse shaping filter has an adjustable centre frequency and pass band, sharp transition with low side lobe level to fit into any FCC mask with least filter length compared to some of the existing techniques which employ optimization techniques(M.Rezaii et al., 2010). 


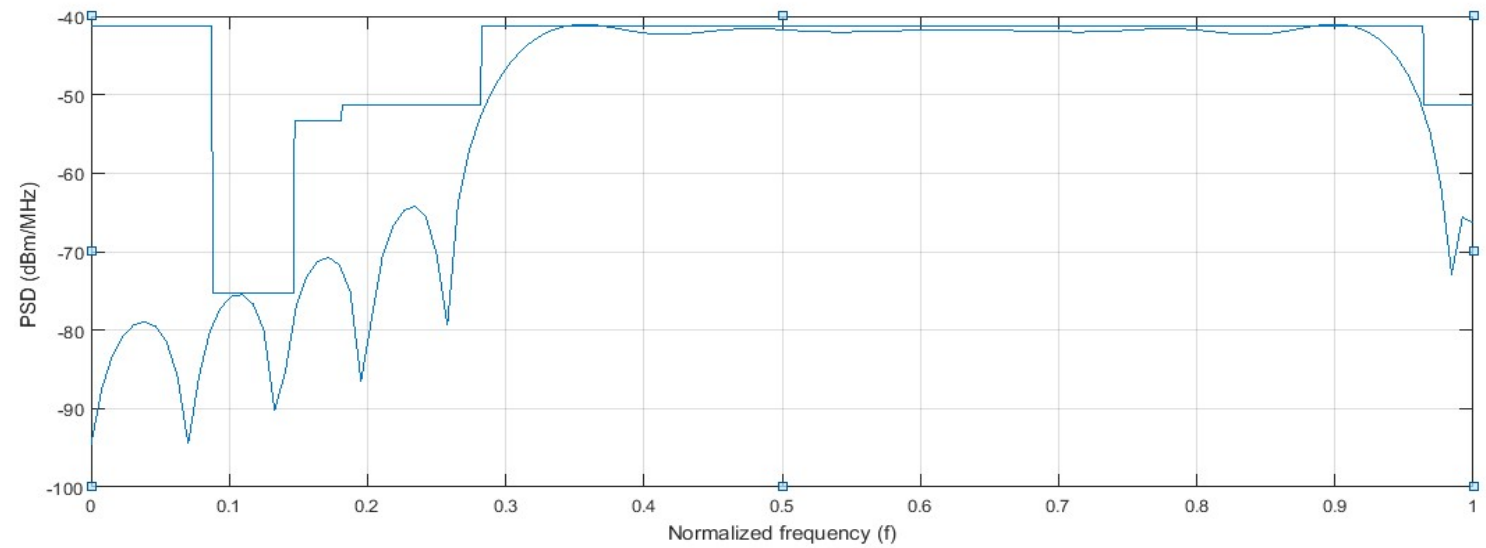

Fig.1: Regulatory FCC Spectral Mask for UWB Band of 3.1to 10.6GHz and PSD of Proposed Band Pass Filter for Pulse Shaping with $L=37$.

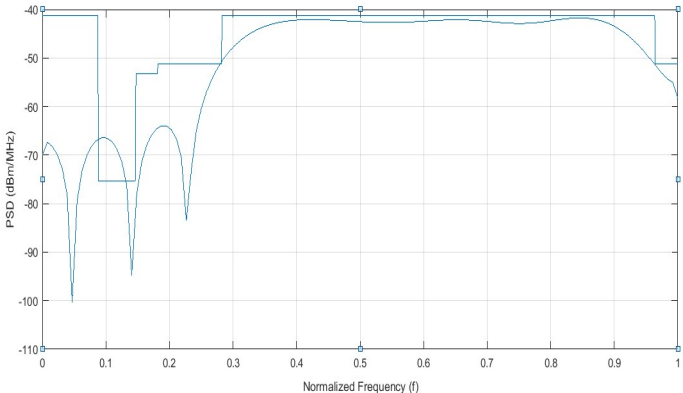

Fig.2: PSD of Proposed Band Pass Filter for Pulse. Shaping with $\mathrm{L}=21$

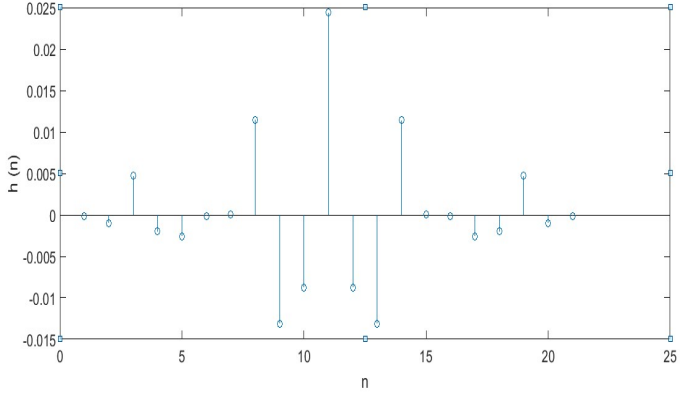

Fig.3. Proposed Pulse Shaper Impulse Response h(n). Shaping with $L=37$

Table 1: Power Efficiency of Proposed Pulse Shaping Method for Filter Lengths $L=37$ and $L=21$ and Other Methods.

\begin{tabular}{|l|l|l|}
\hline Design Technique & Filter lengths L & Power Efficiency \\
\hline Proposed pulse shaping & 37 & $91.2 \%$ \\
\hline Proposed pulse shaping & 21 & $84 \%$ \\
\hline Parks-McClellan (E. Baccarelli et al., 2008) & 37 & $85.47 \%$ \\
\hline SDP-Non Constant PSD(M.Rezaii et al., 2010) & 37 & $92.7 \%$ \\
\hline SDP-Non Constant PSD (M.Rezaii et al., 2010) & 21 & $87.8 \%$ \\
\hline
\end{tabular}

\section{Conclusion}

To avoid interference problems, the FCC has specified the spectral shape for UWB pulse communications. The proposed band pass filter spectrally modifies the UWB pulse to be transmitted. It possesses features of sharp transition to fit the requirements of FCC mask, good stop band attenuation and a simple design procedure without optimization. The pulse shaping filter is adaptable to any change in centre frequency, transition width and pass band width to fit any FCC mask. Expressions for the impulse response are derived, its coefficients are obtained and simulation of pulse shaping band pass filter is carried out. Obtained PSD closely matches with FCC mask and power efficiency of $91.2 \%$ is achieved. The performance using different pulse shaping techniques is examined and compared with the proposed method. 


\section{References}

[1]Y. Rahayu., T. A. Rahman, R. Ngah and P. S. Hall. 2008. Ultra wideband technology and its applications. 2008 5th IFIP International Conference on Wireless and Optical Communications Networks (WOCN '08), Surabaya 1-5.

[2] R. K. Dokania., X. Y. Wang, S. G. Tallur and A. B. Apsel. 2011. A Low Power Impulse Radio Design for Body-Area-Networks. IEEE Transactions on Circuits and Systems I 58: 1458-1469.

[3] HaoluXie et al., 2008. A varying pulse width 5th-derivative gaussian pulse generator for UWB transceivers in CMOS. 2008 IEEE Radio and Wireless Symposium, Orlando, FL 171-174.

[4] Hyunseok Kim ., YoungjoongJoo.2005. Fifth-derivative Gaussian pulse generator for UWB system. 2005 IEEE Radio Frequency integrated Circuits (RFIC) Symposium - Digest of Papers, Long Beach, CA, USA 671674.

[5]G. Zhang, Y. Dai., X. Zhang, Y. Lv and L. Chen. 2011. Design and implementation of UWB pulse with multiple narrow-band interferences mitigation. 2011 International Conference on Consumer Electronics, Communications and Networks (CECNet), XianNing 1154-1157.

[6] M. Z. Win., R.A. Scholtz. 1998. Impulse Radio: How it works. IEEE Communications Letters 2: 36-38.

[7]S. M. Ekome., G. Baudoin, M. Villegas and J. Schwoerer. 2012. Narrowband interference mitigation in UWB communication with energy detector. 2012 IEEE International Conference on Ultra-Wideband, Syracuse, NY $67-71$.

[8] B. A. Lagovsky., A. B. Samokhin and Y. V. Shestopalov. 2016. Pulse characteristics of antenna array radiating UWB signals. 2016 10th European Conference on Antennas and Propagation (EuCAP), Davos 1-3.

[9] B. Parr., ByungLok Cho, K. Wallace and Zhi Ding. 2003 . A novel ultra-wideband pulse design algorithm . IEEE Communications Letters 7: 219-221.

[10] L. Li., P. Wang, X. Wu and J. Zhang . 2011. Improved UWB pulse shaping method based on Gaussian derivatives. IET International Communication Conference on Wireless Mobile and Computing (CCWMC 2011) pp. 438-442.

[11] Hongsan Sheng., P. Orlik, A. M. Haimovich, L. J. Cimini and Jinyun Zhang. 2003. On the spectral and power requirements for ultra-wideband transmission. Proceedings of IEEE International Conference on Communications, 2003. ICC '03., Anchorage, AK 1: 738-742 .

[12]M.Sablatash. 2006. Pulse Shaping, Modulation and Spectrum Shaping for UWB Wireless Communications and the Effects on Interference for Single and Multiband Transmission of UWB Signals.2006 Canadian Conference on Electrical and Computer Engineering, Ottawa 1640-1645.

[13] I.IImmoreev and P. G. S. D. V. Fedotov. 2002. Ultra wideband radar systems: advantages and disadvantages. 2002 IEEE Conference on Ultra Wideband Systems and Technologies (IEEE Cat. No.02EX580), Baltimore, MD 201-205.

[14] H.Werfelli., M. Chaoui, H. Ghariani and M. Lahiani. 2013. Design of a pulse generator for UWB communications. 10th International Multi-Conferences on Systems, Signals \& Devices 2013 (SSD13), Hammamet 1-6.

[15] Y. Wang and L. Zhou . 2008. Performance evaluation in the presence of timing jitter using a novel pulse design based on Hermite functions for UWB communications. 2008 Asia-Pacific Microwave Conference 1-4.

[16] H. Werfelli., M. Chaoui, M. Lahiani and H. Ghariani. 2015. Design of 3.1-5.1 GHz pulse generator for UWB communications. 2015 16th International Conference on Sciences and Techniques of Automatic Control and Computer Engineering (STA) 63-68.

[17] J.Joe. 2003. Cellonics UWB Pulse Generators. International Workshop on Ultra Wideband Systems, Oulu, Finland.

[18] M. Ghavami., L. B. Michael, S. Haruyama, and R. Kohno. 2002. A novel UWB pulse shape modulation system. Journal of Wireless Personal Communications 23: 105-120.

[19] M. Ghavami., A. Amini and F. Marvasti. 2008. Unified Structure of Basic UWB Waveforms. IEEE Transactions on Circuits and Systems II: Express Briefs 55: 1304-1308.

[20] Xiliang Luo., Liuqing Yang and G. B. Giannakis. 2003. Designing optimal pulse-shapers for ultrawideband radios. IEEE Conference on Ultra Wideband Systems and Technologies, 2003, Reston, VA, USA 349-353.

[21] L. A. de Ávila., R. Kunst, E. Pignaton, J. Rochol and S. Bampi. 2016. Energy efficiency evaluation of the pulse shapes and modulation techniques for IR-UWB in WBANs. 2016 IEEE International Conference on Electronics, Circuits and Systems (ICECS), Monte Carlo 173-176.

[22] X. Liu., A.B Prem Kumar, A.S Madhukumar. 2008. Pulse shaping functions of UWB systems. IEEE Transactions on Antennas and Propagation 7: 15121516.

[23] N. C. Beaulieu and Bo Hu. 2006 . A pulse design paradigm for ultra-wideband communication systems. IEEE Transactions on Wireless Communications 5: 1274-1278.

[24] N. C. Beaulieu and B. Hu. 2008. On Determining a Best Pulse Shape for Multiple Access Ultra-Wideband Communication Systems. IEEE Transactions on Wireless Communications 7: 3589-3596. 
[25] Lu Yin and Zhu Hongbo. 2005. UWB pulse design using the approximate prolate spheroidal wave functions. 2005 IEEE International Symposium on Microwave, Antenna, Propagation and EMC Technologies for Wireless Communications, Beijing 1: 450-453.

[26] M. Ghavami., L. B. Michael, and R. Kohno. 2007. Ultra wideband signals and systems in communication engineering, Second edition, John Wiley \& Sons Ltd.

[27] Bo $\mathrm{Hu}$ and N. C. Beaulieu. 2005. Pulse shapes for ultra wide band communication systems. IEEE Transactions on Wireless Communications 4: 1789-1797.

[28] L. J. Gudino., S. N. Jagadeesha and J. X. Rodrigues. 2008. A new filter design for uniform linear array. 2008 5th International Multi-Conference on Systems, Signals and Devices, Amman 1-3.

[29] L. J. Gudino., S. N. Jagadeesha and J. X. Rodrigues. 2009. A novel filter design for spatially interpolated beamformer. 2008 International Symposium on Intelligent Signal Processing and Communications Systems, Bangkok 1-4.

[30]E. Baccarelli., M. Biagi, C. Pelizzoni and N. Cordeschi.2008. UWB Pulse Shaping under Spectral and Synchronization Issues. 2008 IEEE Sarnoff Symposium, Princeton, NJ 1-5.

[31] M. Rezaii. 2010. UWB pulse shaping by FIR filter to enhance power efficiency. IEEE 5th International Symposium on Wireless Pervasive Computing 2010, Modena 522-527.

[32] L. J. Gudino and J. X. Rodrigues. 2008. Linear phase FIR filter for narrow-band filtering . International Conference on Communications, Circuits and Systems, Fujian 776-779. 